\title{
LA CONSTRUCCION DE ESQUEMAS: UN MODELO EXPLICATIVO DE CONSTRUCCION HUMANA
}

\author{
PILAR AZNAR MINGUET \\ Universidad de Valencia
}

Desde el alternativismo constructivista, se ha venido enfatizando el proceso activo del sujeto en la élaboración e interpretación de información, lo cual ha llevado a establecer como postulado básico el hecho de que la construcción de la realidad es un proceso activo y personal.

Esta particular explicación de la configuración bumana a través de la acción surge como reacción a la explicación conductista por la grave carencia que suponia, desde ésta, el hacer depender la configuración conductual de la relación funcional entre estímulos y respuestas; en esta concepción se desdeñaba el papel que desempeñaba el organismo como conductor y difusor del estímulo. La reacción que fue generando tal consideración condujo en un primer momento a introducir en el concepto de conducta aspectos tales como: propositividad consciente, construcción o circularidad retroactiva (Mayor, G. 1985), lo cual produjo dos consecuencias importantes, tales como que la conducta dejó depender del estímulo y/o del organismo y que éste, como concepto, fue desplazado por el sujeto, convertiéndose asi en el protagonista de la conducta. Y fue a partir de aquí cuando empezó a considerarse que el sujeto a través de su acción es quien define las situaciones estimulares, quien transforma y manipula los datos del estímulo y sus estados internos y quien selecciona, planifica, ejecuta y controla la respuesta; todo ello propicia una orientación de la conducta hacia un objetivo o meta, lo cual implica positividad y reflexividad consciente.

Estas nuevas consideraciones representan el núcleo central del cognitivismo, uno de cuyos paradigmas dominantes es la perspectiva el procedimiento de la información.

Las versiones explicativas acerca de como se produce este procesamiento de la información son múltiples; valgan como ejemplos: la teoría de las organizaciones formales de $A$ usubel (1982), la teoría de la equilibración de Piaget (1969, 1973, 1975, 1978), la teoría de los constructos personales de Kelly (1966), la teoría constructivista de Brunner (1978), la teoría de la "setmatriz" de Castillejo $(1981,1983) \ldots$,.. entre otros. 
De todas ellas se puede abstraer la idea general de que el hombre parte de unos principios o postulados básicos a partir de los cuales va percibiendo, comprendiendo y construyendo la realidad; tales principios o postulados han sido "bautizados" con distintos nombres, pero, y aunque con ligeros matices diferenciales, con similar significación; en un entretenido paseo por la literatura "ad hoc" nos hemos encontrado con los siguientes: "scripts" (Abelson, 1981), "estructure" (Brunner, 1963), "inclusores" (Ausubel, 1982), "skills" (Anderson, 1982), "frame" o marco (Minsky, 1975) "plan" (Schank, 1979), "set" (Castillejo, 1981, 1983).

La característica común de todos ellos es la de actuar como una unidad operativa básica del sistema humano que representa el esqueleto o esquema unitario desde el cual se actúa. En la definición de estos "esquemas" (Rumelhart, 1980) hay como una especie de consejo semántico confluyente en entenderlos como constructos básicos desde los cuales el sujeto categoriza, describe, interpreta y decide tanto las propias conductas como las situaciones de interacción social y sucesos sociales.

Los antecedentes que proporcionaron la fuerza explicativa que tiene hoy la noción de esquemas se encuentran a comienzos del $2^{\circ}$ tercio de siglo y más concretamente:

- en los trabajos de Piaget sobre los procesos de pensamiento en los niños (1933), los cuales son explicados en función de la actuación del organismo al entrar en contacto con el entorno, en cuanto que dicha actuación que se operativiza en una serie de acciones, que inicialmente son todas abiertas, pero que se van coordinando rápidamente en series; ésto es lo que conforma lo que él dominó "esquemas", que representan el marco desde el cual el sujeto sigue enfrentándose con su entorno.

- y también en los trabajos de Bartlett (1932) quien concibió la aplicación más importante de los esquemas en su intento por explicar los efectos del conocimiento previo sobre la comprensión y la memoria; según él, el aprendizaje y su significado para el sujeto requiere la disposición en éste de unas estructuras mentales adecuadas, denominadas "esquemas", mediante las cuales el sujeto pone en acción patrones internos con los cuales afrontar nuevas experiencias. Con ello puso de relieve la naturaleza estricta y constructiva de los procesos de la memoria.

\section{La adquisición de esquemas.}

La formación de esquemas se va produciendo a partir de la experiencia personal en situaciones recurrentes (De Vega, M, 1984); está demostrado 
que la generación de los mismos responde a una habilidad básica que se adquiere en etapas muy tempranas del desarrollo madurativo (Piaget, 1933; Nelson, 1981). Sin embargo no han sido aclarados los mecanismos por los que se produce su adquisición; si bien se parte del postulado empírico de que los sujetos tienden a abstraer y almacenar aquellos datos más relevantes en cada situación que se les presenta en su interacción con el entorno; información que pasa a constituir la base del esquema. De esta manera, el sujeto va observando e interpretando el mundo a través de los esquemas que él mismo se va construyendo, desde los cuales da coherencia a los diferentes cursos de acción.

Progresivamente el sujeto va incluyendo en sus propios esquemas la nueva información que recibe, en función de la semejanza o contraste entre las características que definen los elementos que componen los esquemas. Cuando la nueva información no presenta algún tipo de disonancia o discrepancia con la información contenida en el esquema correspondiente, es incluida en éste; pero cuando la disonancia es lo suficiente alta como para no superar los límites de aceptación de la nueva información en el esquema correspondiente, éste, al perder su virtualidad de operar como patrón de ajuste a la realidad, permanece inalterable, propiciándose la construcción de un nuevo esquema. Si la disparidad entre la información nueva y la contenida en el esquema supera dichos límites de aceptación se produce el fenómeno de acomodación, que propicia una modificación del esquema en extensión y contenido, lo cual cumple una función de cambio, reformulación y/o reubicación de esquemas haciedo el sistema de los mismos más comprensivo. Este fenómeno viene explicado desde la teoría de Festinger en cuanto que cualquier estado de discrepancia "tolerable" motiva al sujeto a realizar cambios en su estructura cognitiva tendentes a resolver el estado de desajuste en el se encuentra; dicho desajuste supone una pérdida transitoria del equilibrio de los esquemas que es reajustado mediante la acomodación en los esquemas de la nueva información.

Este proceso constante de desajuste-reajuste va sometiendo a los esquemas a un proceso de diferenciación que propicia la elaboración de nuevos esquemas en virtud de la disgregación "especializada" que va perfilando su contenido de base de base, lo cual explica el incremento progresivo del bagaje y "background" de esquemas, la extensión de contenidos en el mismo, así como la evolución general del sistema constructivo de cada sujeto.

\section{Características de los esquemas.}

El conjunto de esquemas que se va construyendo el sujeto conforma una unidad operativa más amplia o estructura cognitiva entendida como 
una totalidad organizada de esquemas. Las características más definitorias de los mismos son las siguientes:

a) Estar jerarquizádos. La ampliación de los contenidos básicos de un esquema obliga a una ubicación jerarquizada de los mismos a diferentes niveles, conformándose subesquemas cuyo contenido básico está constituido por informaciones más concretas en relación al esquema del que dependen; éste, cuya configuración es más genérica, estaría en la cúspide de la estructura cognitiva actuando como director y guía de los nuevos procesamientos de información en los que los subesquemas actuarían de mediadores. Es decir, que la estructura cognitiva, como sistema de conocimiento humano, estaría formada por un conjunto de esquemas en una disposición jerárqui$\mathrm{ca}$, en la cual se van articulando los esquemas cuyos elementos van conformando subesquemas del mismo.

b.) Estar interrelacionados. Los esquemas no están conformados en compartimientos estancos, sino que forman parte de un sistema interrelacionado, de modo que cuando más abstracto y central sea el esquema, más interrelación guardará con el resto, cumpliéndose así la característica de totalidad de todo sistema, respecto a que cualquier modificación en cualquiera de sus elementos supondrá una reorganización del conjunto; si bien los esquemas más alejados de los referentes conductuales, serán más resistentes a cualquier cambio y por tanto más permanentes y estables.

y por tanto más permanentes y estables.

c) Ser variables y flexibles. La composición básica de esquemas y subesquemas va variando en función de los aportes de nueva información, lo cual les confiere el valor de ser tan flexibles como sea requerido en los contínuos reajustes inherentes a las necesidades de acomodación a la realidad; es decir que los esquemas tienen un cierto grado de permeabilidad que les permite admitir nuevos elementos dentro de su ámbito de competencia, los cuales propician cambios en el sistema jerárquicamente organizado de los mismos. Ello no hace peligrar la consistencia del sistema de esquemas como totalidad organizada en virtud de la permeabilidad de los mismos, que permite una integración de las inconsistencias aparentes o reales a niveles más bajos de abstracción. Precisamente de la permeabilidad de los esquemas dependerá la tolerancia personal a las inconsistencias diarias a las que el sujeto se enfrenta.

d) Ser activados a través de dos modos operativos básicos. La ordenación jerarquizada de los esquemas implica una doble direccionalidad: de supraordenación y de subordenación; esta doble direccionalidad en la circulación de información permite poner en acción a los mismos de dos formas distintas: o bien desde los más generales a los más concretos - de arriba a abajo-, o bien de los más concretos a los más generales - de abajo a arriba-. Estos dos tipos de procesamiento han sido ampliamente analizados por Norman 
(1979), teniendo su punto de partida el $10^{\circ}$ en el esquema de más alto nivel conceptual, desde donde se extiende la activación bacia los subesquemas correspondientes, generando desde ellos nuevos esquemas madiacionales de ajuste a los datos; se trata de un procesamiento controlado, consciente y activado por planes y objetivos internos. El segundo tipo de procesamiento tiene su origen en la propia información contenida en el input, cuyos datos son los que dirigen la actividad procesadora; es más bien un tipo de procesamiento automático que activa directamente desde el estímulo (EYSENCK, M. W. 1984) a los esquemas de nivel inferior; ello explica que, en ocasiones, estos esquemas inferiores sean disparados automáticamente sin un previo ajuste con los esquemas de nivel superior de los que dependen, dando lugar a los llamados "actos fallidos" o equivocaciones (Norman, 1981); estos esquemas de nivel inferior, desde donde se origina el procesamiento de abajo a arriba, opera al margen de la conciencia y se ajustan más estrictamente a las exigencias ambientales. la bondad de uno y de otro tipo ha sido puesta de manifiesto en diversos trabajos, concluyéndose al respecto que los dos sistemas de procesamiento se requieren mutuamente, lo cual ha abierto camino a la hipótesis interaccionista, según, la cual los esquemas van guiando la interpretación de los datos y al propio tiempo dicha interpretación va confiriendo consistencia al esquema en la medida en que lo datos se adecuan al mismo (Arnau Gras, 1985).

e) Estar especializados. Cada esquema representa un modo de enfrentarse a un aspecto particular del entorno; de lo cual se deduce que a cuantos más aspectos diferentes del entorno se enfrente el sujeto, su background de esquemas será mayor.

\section{Funciones de los esquemas.}

A nivel teórico explicativo los esquemas tienen un carácter multifuncional por cuanto su utilidad operativa es enormemente amplia:

- Función constructora del conocimiento-. En cuanto que la adquisición de esquemas responde a un proceso interno que pone en relación constante los fenómenos, datos y estímulos que provienen de la realidad y la conducta que ante ellos manifiesta el sujeto codificándolos e interpretándolos (Piaget, 1978).

- Función selectiva-. Un sujeto no puede atender todos los múltiples y variados aspectos que caracterizan la información que recibe, sino que, en base a sus esquemas, va seleccionando aquellos aspectos considerados relevantes desde los mismos. Es decir, que, al activarse un esquema, éste funciona como un mecanismo de selección atencional, en el sentido de que el sujeto tiende a procesar fundamentalmente aquella información que encaja en dicho 
esquema y a deshechar la información que desde el punto de vista del esquema se considera irrelevante (Kintsch y Van Dik, 1978).

-Función predictiva - Al canalizar los esquemas la codificación, representación, organización y recuperación de la información, generan espectativas de lo que va a acontecer, lo cual le confiere al sujeto una fuerte consistencia comportamental que le permite encarar la incertidumbre del futuro desde la relativa estabilidad del sistema de esquemas que se ha construído; en este sentido funcionan de manera análoga a las teorías científicas, en cuanto que dan lugar a inferencias y predicciones (Rumelhart, 1980)

-Función de control-Dado que los esquemas son representaciones que posee una persona sobre un aspecto de la realidad, ello posibilita el establecimiento de metas y la planificación secuencializada de acciones adecuadas a las mismas; en dicha planificación se inscriben todo tipo de patrones conducturales, incluso los más complejos.

-Función de guía y orientación en los procesos de comprensión - Los datos e información nueva contenida en el input es interpretada desde los esquemas y subesquemas correspondientes identificando y categorizando objetos y situaciones, confiriéndoles así un concreto y específico grado de significación; es decir que el conjunto de esquemas construídos por el sujeto actúa como marco asimilador al atribuir significaciones al mundo que le rodea, actuando así como instrumentos de comprensión de la realidad. De esta manera, la atribución de significación a los nuevos datos e informaciones consiste en integrarlos desde los esquemas ya elaborados en el marco de asimilación disponible (De Vega, 1984).

-Función evaluadora- Desde los esquemas se evalua el grado en que se interpreta de forma adecuada y pertinente la información sensorial y el grado de calidad de su propio ajuste a dicha información; es decir que los esquemas, integrados en la estructura cognitiva se convierte en ejes en torno a los cuales se van evaluando las nuevas informaciones.

\section{Tipos de esquemas}

Los esquemas que se va construyendo el sujeto presentan fundamentalmente una doble naturaleza que da pie a poder hablar de dos tipos de esquemas:

- Los esquemas de acción.

- Los esquemas de conocimiento.

Los primeros se construyen "espontáneamente", dando lugar a determinadas formas de interacción entre el sujeto y la realidad que son cada vez más ajustadas en cuanto que aquí, el sujeto, puede comprobar "sobre la marcha" los resultados, positivos o negativos, en la eficacia de este tipo 
de esquemas. Estos esquemas son los primeros instrumentos de comprensión de la realidad que rodea al sujeto, siendo, desde la teoría genética, explicados como organizaciones de acciones que son mantenidas conservadas por el niño a través de las situaciones y objetos a los que se aplican. Van referidos a un tipo de estructura de conocimiento no conceptual, sino operativa o ejecutiva; es decir dirigida a la acción de ahí que tengan un carácter netamente procedimental. Fundamentalmente vienen careacterizados por: activarse en función de las intenciones del sujeto (Norman, 1981), incluir secuencias de acción motrices para alcanzar la meta (Abalson, 1981) y estar generados por los datos provinientes de la información ambiental, lo cual lleva a la activación de esquemas mediacionales. Por ejem: si quiero ir al cine, es mi intención de ir, lo que activa el esquema correspondiente que, a su vez, me permite desencadenar y al propio tiempo controlar la operativización de un conjunto de esquemas de acción subordinados al 1. ${ }^{\circ}$, es decir, mediadores entre él y la meta a alcanzar; estos esquemas subordinados van dirigidos a la consecución de objetivos instrumentales necesarios a la consecución de la meta del esquema del que dependen; siguiendo el ejemplo puesto: salir a la calle, coger el autobus, sacar la entrada...

La puesta en acción repetida de este tipo de esquemas conlleva a una "babituación" que les confiere un alto grado de automatismo, bastando que se active la intención de alcanzar la meta general (ir al cine) para que las acciones correspondientes se ejecuten "sin apenas consumo atencional" (De Vega, M. 1984).

Así como los esquemas de acción están especializados en la conducta, los esquemas de conocimiento son los que más netamente contienen información conceptual sobre la realidad, aunque también disponen de componentes procedimentales, Responde a un procesamiento de la información girado conceptualmente y desempeñen un papel básico en la utilización de los sistemas de percepción y memoria; pueden incluirse en los mismos: los "esquemas visuales" o "marcos" según la consideración de Minsky (1975); los "esquemas sociales", que pueden hacer referencia a: prototipos de personas (estudios, liberal...), o temas de roles (profesor, arquitecto...), o temas referentes al tipo de relaciones sociales establecidas (compañero, opositor, amigo...), o temas actitudinales ante la vida (como: ser liberal, conservador, luchar por la libertad o para contrarrestar las desigualdades sociales...) y en los cuales se dan fuertes componentes ideológicos y de sistemas de creencias; Son esquemas de acción; los "esquemas situacionales" o "guiones" (scripts) según la consideración de Abelson (1981); Según los cuales las personas tenemos, modos de comportarnos en los distintos contextos, de comunes acuerdo a formas socialmente esclarecidas o escenarios conductuales (behasionel sittings en terminología de Banker) en los que nos relacionamos, conferencias, biblioteca, supermercado... Nuestra conducta en estas 
situaciones esté controlada y disipada por los acontecimientos del contexto -guiada por los datos-. De esta manera, los "guiones" representan una estructura fundamental, para codificar y recordar una determinada actividad (ir a una Conferencia, al cine...). lo "esquemas autoconceptuales" referentes al conocimiento más o menos articulado que cada persona posee sobre sí misma (Markus, H. 1977) sobre sus actitudes, metas, comportamiento, sexo...

Cabe citar por último a los que se podrían denominar "esquemas cien. tíficos" entendidos como un proceso de apropiación del conocimiento que tiene lugar en las situaciones de enseñanza aprendizaje; básicamente son de naturaleza instructiva y su adquisición, por parte del sujeto viene fomentado desde el propio curriculum.

En resumen podemos decir que el modelo de esquemas como teoría explicativa acerca de cómo el hombre procesa la información que recibe es, en el estado actual de la cuestión, un modelo en construcción; sin embargo, su poder altamente explicativo, respecto a la validez de las representaciones esquemáticas para todo tipo de áreas de conocimiento, le confiere un grado de plausibilidad científica que está siendo ampliamente aceptado. 


\section{BIBLIOGRAFIA}

ABELSON, R.P. (1981) "Psychological status of the script concept". American Psychologist, 36, n. 7, págs 715-729.

ANDERSON, J.R. (1982) "Acquisition of cognitive skill". Psichological Revierw, vol. 89,n." 4.

ARNAU GRAS, J. (1985) "Procesamiento en prosa y estructuras cognitivas" en MAYOR, J. Actividad bumana y procesos cognitivos. Alhambra. Granada.

AUSUBEL, D.P. (1982) Psicología educativa. Un punto de vista cognitivo. Trillas. Barna.

BARTLETT, F.C. (1932) Remembering. A study in experimental and social Psychology. Cambridge. University Press.

BRUNNER, J. (1963) El proceso de la educación. Uthea, México. - (1978) El proceso mental en el aprendizaje. Narcea.

CASTILLEJO, J.L. (1981) "Modelo funcional de la educación" en CASTILLEJO, J.L., ESCAMEZ, J., y MARIN, R. Teoría de la educación. Madrid. Anaya.

- (1983) "La educación como elaboración de consistencias" en Varios: Teoría de la edu. cación (El problema de la educación). Límites. Murcia.

DE VEGA, M. (1984) Introducción a la psicología cognitiva. Alianza. Madrid.

EYSENCK, M.W. (1984) $A$ handbook of cognitive Psychiligy. LEA. Londres.

KELLY, G.A.: Teoría de la personalidad. La psicología de los constructos personales troquel.

KINTSCH, W. y DUK, T.A. (1978) "Toward a model of text comprehension and production". Psychological Review, 85'5.

MARKUS, H. (1977) "Self-Schemata and processing information about the self". Journal of personality and social Psyhology. vol 35, n. 2, pags 63-78;

MAYOR, J. (1985) Actividad bumana y procesos cognitivos. Alhambra. Granada.

MINSKY, M. (1975) "A framework for representing knwoledge" en Winston P.H. The psychology of computer of computador visión. Mx Graw-Hill. New York.

NORMAN, D.A. y BORROW, D.G. (1979) "Sobre el papel de los procesos activos de la memoria en la percepción y cognición" en COPER, C. H. Estructura de la memoria bumana Omega. Barna.

NORMAN, D.A. (1981) “Categorization of action slips”. Psychological Review, vol, 88, n." 1 pags. $1-15$.

NELSON, K (1981) "Social cognition in a script framework" en Social cognitive development. FLAVELL y ROSS. Cambridge University Press.

PIAGET, J. (1933) La representación del mundo en el niño Espasa-Calpe. Madrid. - (1969) Biología y conocimiento. Siglo XXI. Madrid.

- (1973)Psicología y Pedagogía. Ariel. Barcelona.

- (1975) Psicología de la inteligencia. Psiche. Buenos Aires.

- (1978) La equilibración de las estructuras cognitivas. Problema central del desarrollo. Siglo XXI. Madrid.

RUMELHART, D.E. (1980) "Schemata: the building blocks cognition", en SPIRO: Theoretical issues in reading comprebension. New York. Eribaum Assoc.

SCHANK, R.C. (1979) "El papel de la memoria en el procesamiento del leguaje", en COPER, Ch. N.: Estructura de la memoria bumana. Omega. Barcelona. 
